\title{
SERVICE DE LUXE ET CLASSES SOCIALES
}

Gabriele Pinna et Bertrand Réau

Le Seuil | «Actes de la recherche en sciences sociales »

2011/5 n 190 | pages 72 à 77

ISSN 0335-5322

ISBN 9782021064216

Article disponible en ligne à l'adresse :

https://www.cairn.info/revue-actes-de-la-recherche-en-sciencessociales-2011-5-page-72.htm

Distribution électronique Cairn.info pour Le Seuil.

(c) Le Seuil. Tous droits réservés pour tous pays.

La reproduction ou représentation de cet article, notamment par photocopie, n'est autorisée que dans les limites des conditions générales d'utilisation du site ou, le cas échéant, des conditions générales de la licence souscrite par votre établissement. Toute autre reproduction ou représentation, en tout ou partie, sous quelque forme et de quelque manière que ce soit, est interdite sauf accord préalable et écrit de l'éditeur, en dehors des cas prévus par la législation en vigueur en France. Il est précisé que son stockage dans une base de données est également interdit. 


\title{
par Gabriele Pinna et Bertrand Réau
}

\section{Service de luxe et classes sociales}

\author{
À propos de Rachel Sherman, Class \\ Acts: Service and Inequality in Luxury Hotels, \\ Berkeley/Los Angeles, \\ University of California Press, 2007.
}

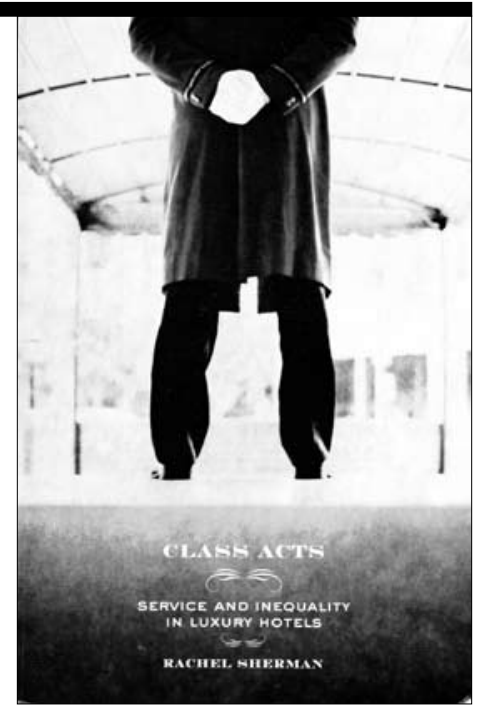

Rachel Sherman, sociologue américaine, Assistant Professor à la New School for Social Research de New York, a principalement travaillé sur l'hôtellerie de luxe et le syndicalisme aux États-Unis. Sa démarche et ses références théoriques s'insèrent dans le courant de la sociologie critique américaine, d'inspiration interactionniste et néo-marxiste. Elle a mené durant un an une enquête par observation et par entretiens dans deux hôtels de luxe aux États-Unis ${ }^{1}$. Son intérêt pour l'hôtellerie de luxe naît au cours d'une collaboration entamée dans les années 1990 avec un syndicat de salariés de l'hôtellerie-restauration. Pour commencer son enquête, elle réalise des entretiens ${ }^{2}$ avec les managers des hôtels, ce qui lui permet de négocier son accès au terrain : elle obtient la possibilité de travailler dans deux hôtels sur plusieurs postes (femme de chambre, réceptionniste, concierge, etc.). Cela lui a donc permis de côtoyer à la fois les clients et les salariés et d'étudier les différentes situations de travail qui peuvent se présenter dans le déroulement quotidien d'une journée hôtelière.

L'ouvrage est composé de six chapitres : le chapitre 1 porte sur le service de luxe en tant que produit et s'appuie largement sur une analyse du discours du management et de la littérature en matière de marketing de services de luxe. Le chapitre 2 décrit l'organisation du travail dans les hôtels. Le service de luxe, fort complexe en raison de la variabilité des demandes des clients, est mis en place à travers une organisation du travail qui prévoit un certain degré d'autonomie pour le personnel de l'hôtel (notamment pour les salariés du " front office " en interaction avec les clients). Le chapitre 3 met en avant l'engagement actif des salariés à travers une description minutieuse des « jeux » auxquels ils participent afin d'y « trouver leur propre compte ». Cela permet un renforcement de leur identit personnelle et professionnelle. Elle se réalise aussi grâce aux comparaisons que les salariés font entre leur propre travail et celui de leurs collègues. Par ailleurs, les salariés élaborent des systèmes de classement qui ne portent pas exclusivement sur le pouvoir économique des clients. Ils peuvent parfois se considérer supérieurs aux clients (chapitre 4). Le chapitre 5 se concentre sur les mécanismes de la réciprocité qui favorisent l'essor de relations individualisées entre clients et salariés : les clients, en effet, remercient les salariés, laissent des pourboires et en général s'adressent aux salariés très poliment. Enfin, le chapitre 6 montre comment ces interactions entre les clients et les salariés dans l'hôtellerie de luxe contribuent à naturaliser la domination et à légitimer les droits des clients dans un milieu de luxe.

\section{Introduire les classes sociales} dans l'étude de la relation de service

À partir d'une analyse du travail effectivement réalisé par l'ensemble des salariés et des interactions entre les clients et les salariés, Sherman introduit la question des relations

\footnotetext{
1. L'auteure propose aussi une analyse de la littérature marketing et de gestion portant sur ce type d'hôtels.

2. Au cours de sa recherche, l'auteure réalisa aussi des entretiens avec des salariés et 19 entretiens avec des clients de l'hôtellerie de luxe. Pour autant, l'angle d'approche privilégie les salariés, les clients ne sont étudiés qu'en relation avec eux. Leurs profils ne sont guère détaillés.
} 
entre les classes sociales dans l'univers des services. Selon l'auteure, la problématique de l'exploitation dans les services, surtout dans ceux qui, comme les hôtels de luxe, comportent une distance sociale élevée entre les salariés et les clients, ne peut pas être traitée de manière analogue à celle adoptée traditionnellement dans des recherches concernant le secteur industriel.

Les approches classiques ont qualifié d'« exploitation de classe » le moment du travail productif des ouvriers dans les ateliers, et ils ont recherché des traces de leur aliénation ou résistance dans ces lieux de travail. Mais, pour Sherman, dans les hôtels de luxe, l'enjeu n'est pas seulement l'exploitation des salariés, mais aussi l'acceptation de la part de ces mêmes salariés d'un système de distribution inégal des droits entre eux et les clients. Ainsi, les clients sont légitimés à recevoir non seulement un travail matériel important de la part des salariés, mais aussi un " travail émotionnel ${ }^{3}$ ", au sens où les salariés, notamment ceux en interaction avec les clients, doivent garder une gentillesse déférente et "spontanée » dans n'importe quelle situation de travail. Quelles sont alors les raisons qui rendent acceptable cette inégalité aux yeux des salariés?

D'après Sherman, la production et la consommation du service de luxe " normalisent " et " banalisent " les inégalités de classe, considérées comme allant de soi pour les salariés de ces hôtels. La naturalisation de l'inégalité est la conséquence paradoxale des stratégies mises en œuvre par les salariés afin de valoriser leur identité au travail par la recherche de l'autonomie, de l'acquisition de compétences ou du prestige associé à leur poste de travail.

En citant Marx, Sherman rappelle comment l'obscurcissement de l'exploitation dans la production industrielle se passe en dehors du cadre du travail, puisque lorsque les marchandises sont échangées dans le marché, les relations de domination entre les hommes assument la forme illusoire d'une relation entre des choses, par la médiation de l'argent. Dans les hôtels de luxe, l'inégalité ne peut être cachée en aucune manière. Pour autant, en amenant les clients et les salariés à considérer leurs relations comme " individuelles ", les interactions voilent leur nature de classe. La personnalisation du service renforce l'individualisation des relations. Elle renforce le sentiment de singularité chez les clients alors que, de leur côté, les salariés cherchent également à valoriser leur singularité.

Les hôtels de luxe ne se limitent pas à confirmer l'inégalité de classe. Au contraire, ils exercent une fonction de reproduction sociale de la même manière que l'école, la famille ou les institutions religieuses. Selon l'auteure, il faut réexaminer les oppositions entre production et culture, contrôle et résistance, salariés et clients. Dans ce sens, les relations de production-consommation des services seraient des « relations culturelles ", et l'acceptation de l'inégalité dans le lieu de travail de la part des salariés fait partie d'une vision plus générale du monde social. En effet, pour Sherman, le luxe prend la forme d'une croyance religieuse : une idéologie qui attribue aux individus leur place dans la société. Cette reconnaissance implicite d'une position de domination et de la légitimité des clients à consommer les services de luxe est considérée par Sherman comme un moment spécifique dans la création de l'habitus. L'hôtel est donc une institution qui produit et reproduit ces dispositions de classe, notamment cette prérogative des classes dominantes qui consiste à se sentir chez soi dans les palaces et à en maîtriser les codes de conduite, mais qui ne se limite pas à reproduire le statu quo. Service accessible à n'importe quel client capable d'en soutenir le prix, l'hôtel de luxe paraît aussi jouer un rôle important dans les processus de légitimation des «nouveaux riches ». Les parvenus lorsqu'ils descendent dans un hôtel de luxe ne se limitent pas à consommer un service : ils appréhendent les codes de conduite à respecter dans le hall d'un hôtel de luxe. Les salariés y jouent paradoxalement un rôle important : en effet, eux-mêmes disposent du pouvoir de censure sur les clients qui ne maitrisent pas les attitudes et les comportements attendus dans ce milieu social.

\section{Le service de luxe et l'organisation du travail}

Le service de luxe est caractérisé par une forte personnalisation des prestations qui, entre autres choses, permet de fidéliser la clientèle : il s'agit pour les salariés d'anticiper, de trouver une solution pour n'importe quel désir ou besoin ; de fournir un travail physique "illimité »; et de se montrer déférents et sincères envers les clients ${ }^{4}$.

Pour personnaliser le service, il faut appeler les clients par leur nom et, si possible, se rappeler les prénoms de leurs enfants ou de leurs animaux de compagnie. Les salariés doivent ensuite s'efforcer d'individualiser leurs conversations avec les clients en mobilisant les informations dont ils peuvent disposer (notamment les activités que les clients ont pu accomplir lors d'un précédent séjour). Enfin, le service de luxe exige que tous les salariés (même ceux qui, comme les femmes de chambre, ne sont pas en interaction directe avec les clients) observent les préférences des clients, lesquelles peuvent être d'ailleurs classées dans des bases de données.

L'observation des goûts et des préférences des clients de la part du personnel de l'hôtel se situe à deux niveaux : le premier consiste à enregistrer toutes les préférences

3. Arlie Russell Hochschild, The Managed Hearth: the Commercialization of Human Feeling, Berkeley, University of California Press, 1983.

4. Depuis la fin des années 1980 , le service est au centre du discours des managers au-delà de l'hôtellerie de luxe. 
exprimées par les clients, le second, plus subtile, exige une observation fine de leur comportement afin de pouvoir les surprendre par des services devançant leurs demandes. Ces outils garantissent au client la reconnaissance de sa singularité. Et plus les clients sont des " habitués ", plus les hôtels disposent d'informations permettant cette personnalisation du service. Le client est accueilli par son nom, les employé(e)s chargé(e)s de l'accueillir ou même les managers dans le cas d'un client particulièrement important s'adressent à lui comme s'il était un « ami » à qui l'on demande des nouvelles. La satisfaction des « besoins » du client va jusqu'à l'anticipation de ce qu'il " désire " implicitement, des demandes qu'il n'a pas formulées explicitement ou dont il n'a même pas conscience. Ce processus peut créer des désirs, en assurant des services que les clients ne savent même pas vouloir.

La reconnaissance du client passe aussi par une réponse rapide et efficace aux problèmes qu'il exprime. Les managers demandent, lors des séances de formation du personnel (auxquelles Sherman a participé), qu'il obtienne tout ce qu'il désire. Les salariés doivent lui apporter une réponse compréhensive, se montrer concernés par ses difficultés et, en cas de récrimination contre la qualité des prestations, commencer par s'excuser. Ils doivent se montrer sensibles aux problèmes qu'il exprime, sans quoi le service rendu est considéré comme un échec. L'impératif de « jamais dire non à un client » implique que celui-ci puisse, par certains de ses désirs, transgresser les règles établies, mais il s'agit là d'un élément structurant du service dans les hôtels de luxe par opposition aux hôtels de moyenne gamme.

Les clients peuvent profiter des bénéfices du travail « illimité » des salariés, lequel n'est pas seulement physique, mais aussi émotionnel. L'auteure propose d'appeler les services de ces hôtels des "démonstrations de travail ", qui impliquent le travail humain visible ou de simples marqueurs de travail. Pour ce qui concerne le travail visible, comme Veblen ${ }^{5}$ l'avait montré, dans le milieu de luxe, le personnel est censé réaliser des tâches hautement symboliques : les « démonstrations de travail » physiques rendent le luxe plus somptueux. Les salariés accomplissent beaucoup de tâches afin d'éviter tout travail physique aux clients (comme prendre en charge leurs bagages ou garer leurs voitures). On préfère par exemple affecter un salarié à ouvrir et fermer la porte d'entrée du hall au lieu d'y installer une porte automatique.

Par ailleurs, les marqueurs du travail invisible des salariés montrent que, même en l'absence des clients, une activité continue vise à prendre soin d'eux. Par exemple, dans l'un des hôtels étudiés, les clients reçoivent chaque matin leur journal dans un joli sac, soigneusement accroché à la poignée de la porte, où il est marqué : "bonjour ». Les salariés doivent exprimer leur plaisir à réaliser les tâches demandées et sont formés pour employer de manière récurrente certaines expressions : "c'est un plaisir pour moi ", " à votre service ", " n'hésitez pas à m'appeler en cas de besoin ", " je vous en prie ", etc. Ne pas respecter ces standards peut engendrer des plaintes de la part des clients. La marque distinctive des hôtels de luxe, par rapport aux autres établissements, réside dans le travail, visible ou invisible, dans la rapidité et l'excellence du service et dans la manière dont celui-ci est accompli ; chaque salarié doit en effet se montrer déférent, souriant, enthousiaste dans la réalisation de son travail, contrôler son apparence, autoriser les clients à entamer et à interrompre une conversation, accepter de se faire appeler par son prénom.

La diversité des clients et des situations de travail contraint les managers à laisser aux salariés l'autonomie indispensable pour qu'ils élaborent sans gêne des réponses pertinentes, tout en respectant les codes et les standards du luxe. Mais ces principes peuvent être mis en œuvre par des organisations de travail différentes. L'auteure identifie le Luxury Garden à une organisation de type « hiérarchique professionnel » : le service offert y est plus formel et professionnel ; l'autonomie et l'indépendance des travailleurs sont renforcées par le discours du manager qui encourage le sentiment d'appartenance à l'organisation. Au Royal Court, en revanche, prévaut une organisation de type " flexibilité informelle ": les relations y sont plus chaleureuses et plus souples, mais la qualité du service est inférieure aux standards de l'hôtellerie de luxe ; la division et la spécialisation moins marquées du travail s'accompagnent d'une difficulté, pour les salariés, à développer un sentiment d'autonomie.

Pour autant, dans les deux hôtels, il y a une division du travail entre les salariés « visibles " en interaction avec les clients (comme les réceptionnistes et les concierges) et ceux " invisibles ", comme les femmes de chambre, qui disposent d'une moindre autonomie. Cette division est aussi raciale et sexuée : les salariés "invisibles ", chargés du " sale boulot ", mais peut-être moins touchés par le rapport de subordination aux clients, sont, dans les deux hôtels, des femmes immigrées d'origine hispanique, asiatique ou afro-américaine. Les clients ne récompensent que très rarement leur travail par des pourboires. Ce sont plutôt les concierges, les bagagistes, les voituriers ou les grooms (autrement dit, les salariés actifs sur la " scène " au sens de Goffman) qui en bénéficient. Cela accroît encore l'invisibilité du travail des salariés du " back office ", comme les femmes de chambre. Pour décrire le travail des salariés qui jouent sur la « scène » des hôtels de luxe, Sherman propose la métaphore goffmanienne du « service théâtral ». Comme le théâtre, l'hôtel de luxe dispose d'une scène et de coulisses

5. Thorstein Veblen, Théorie de la classe de loisir, Paris, Gallimard, 1970 [1899]. 
qui sont à leur tour subdivisées, ou encore, comme les théâtres, les hôtels sont ouverts aux publics qui peuvent se permettre de payer le billet d'entrée, et ils dépendent, pour leur succès, des commentaires des professionnels et des spectateurs. La performance qui se réalise sur la scène constitue un moment important de la production et de la consommation simultanée du service offert ${ }^{6}$. D'où l'importance du « travail émotionnel » et de la prestation des salariés pour le bon déroulement du service de luxe.

\section{Engagement actif des salariés et normalisation de l'inégalité}

Lorsque Sherman commence à travailler à l'hôtel Royal Court, elle est surprise de ne pas y trouver de salariés mécontents des tâches exigées. Au contraire, au lieu d'exprimer du ressentiment ou un sentiment d'aliénation, les salariés sont engagés dans leur travail et semblent l'apprécier. D'après Sherman, si les salariés de l'hôtellerie racontent souvent des histoires de demandes jugées scandaleuses ou soulignent les prix très élevés des chambres, ils considèrent les droits de la clientèle comme un signe naturel de la qualité offerte par l'hôtel. Les critiques et les moqueries à l'encontre de quelques clients ne renvoient jamais explicitement à des différences de classe et le système n'est pas ouvertement remis en cause. L'écart entre les positions sociales des salariés et des clients, l'obligation pour les salariés de se subordonner aux clients, apparait comme un simple fait établi.

Ainsi Sherman emprunte à Burawoy ${ }^{7}$ le concept de " consentement " qu'il mobilise dans ses travaux sur la production dans l'industrie. Pour Burawoy, les salariés qui possèdent un peu d'autonomie deviennent engagés dans leur travail en reprenant à leur compte les objectifs de production valorisés dans leurs ateliers. Par le consentement à l'exercice du travail, les salariés légitiment sans le vouloir les conditions de l'appropriation de leur force de travail par l'entreprise. Sherman utilise également l'idée de « normalisation ", qui se réfère à la banalisation de l'inégalité du rapport social entre les salariés et les clients (en termes de droits et de responsabilités). Dans ce cadre, la critique ne peut être formulée qu'à titre individuel, sans jamais toucher aux relations collectives entre les deux groupes sociaux.

Dès lors, le " consentement " et la " normalisation » sont les éléments qui permettent aux salariés de se représenter comme indépendants et non subordonnés vis-à-vis des managers, des collègues et des clients ${ }^{8}$. Cela passe par la pratique de certains jeux, parfois le sentiment de supériorité vis-à-vis de leurs collègues ou des clients, ou encore par des relations privilégiées avec certains clients qui leur donnent l'impression de parler d'égal à égal9 .
Les stratégies complexes adoptées par les salariés et par les clients contribuent à individualiser les relations entre ces deux groupes sociaux. Les clients représentent des véritables « agents de production ", au sens de Burawoy, puisque leurs actions et leurs désirs (par exemple le type de chambre qu'ils préfèrent ou leur propension à laisser des pourboires) contribuent à rendre imprévisibles les jeux liés au contrôle de la production et des efforts. Les membres du "front desk » (concierges et réceptionnistes) gèrent les aléas concernant les réservations, les prix des chambres ou les réservations aux restaurants. Ces activités fluctuantes témoignent de l'imprévisibilité du volume de travail à un moment donné à l'hôtel. Les salariés peuvent profiter de cette situation floue, qui échappe à la programmation du manager. Ils cherchent ainsi à contrôler leur propre temps de travail (cadences), à respecter des standards du luxe tout en se protégeant vis-à-vis des plaintes des clients. Pour ce faire, ils essaient de prévoir et de classifier les comportements des clients en les catégorisant. L'observation des clients est donc aussi un outil de protection pour les salariés.

Le " jeu des pourboires ${ }^{10}$ ", auquel seuls les salariés en contact direct avec les clients peuvent participer, est, de tous les jeux pratiqués dans l'hôtel, celui qui permet

6. Le salaire horaire net du personnel de l'hôtel est variable en fonction du poste de travail. Les pourboires constituent une intégration importante du revenu, mais les clients en laissent davantage aux salariés du " front office ". Au Luxury Garden, le salaire horaire d'un réceptionniste et d'un concierge est de $15 \$$, mais le concierge reçoit environ 500 \$ de pourboires par semaine alors que les réceptionnistes n'en ont pas. Pour ce qui concerne la division du travail, d'après Sherman, les réceptionnistes peuvent être indifféremment des hommes ou des femmes, américains (blancs) ou immigrés (hispaniques, chinois et philippins). Les concierges (hommes ou femmes) sont davantage américains (blancs ou d'origine asiatique). Les standardistes sont en prévalence des femmes d'origine hispanique. Leur salaire horaire est de 14 \$ et, n'ayant pas d'interactions directes avec les clients, elles ne reçoivent pas de pourboires. Les agents de réservations (hommes ou femmes) sont américains (blancs ou d'origine asiatique) et ils reçoivent un salaire horaire de 14 \$ plus des commissions sur la vente des chambres. Le portier est un métier essentiellement masculin, apanage d'hommes américains (blancs) ou d'immigrés (chinois). Le salaire horaire est de 10 \$. Parmi les salariés de l'hôtel, les portiers reçoivent le plus de pourboires, environ 150 \$ pour chaque journée de travail. Le bagagiste est un métier masculin : les salariés sont américains (blancs) ou immigrés (philippins, chinois et hispaniques). Ils reçoivent environ $100 \$$ de pourboires à chaque journée de travail. Les femmes de chambre sont normalement chinoises et leur salaire horaire est de $13 \$$. Elles ne reçoivent de pourboires que très rarement. Enfin, les employés du room service - immigrés de l'Europe de l'Est - reçoivent un salaire horaire de $10 \$$. Leurs pourboires correspondent au $15 \%$ du prix (le service n'est pas compris dans le prix aux États-Unis). Les salaires et les pourboires sont plus bas à l'hôtel Royal Court et le nombre de salariés immigrés dans cet hôtel est plus important que dans l'hôtel Luxury Garden.

7. Voir l'article de Pierre Fournier, "Deux regards sur le travail ouvrier : à propos de Roy et Burawoy, 1945-1975 ", Actes de la recherche en sciences sociales, 115, décembre 1996, p. 80-93.

8. Sherman essaie de dépasser les dualismes théoriques d'authenticité et de performance, d'engagement et de passivité au travail, qui marquent la littérature sur les services aux États-Unis, et montre comment les salariés en interaction avec les clients s'appuient sur un ensemble de stratégies complexes et souvent contradictoires, à travers lesquelles ils construisent finalement leur propre identité, en se définissant comme forts et importants.

9. Ces stratégies ne sont d'ailleurs pas nécessairement intentionnelles. 10. P. Fournier, "Deux regards sur le travail ouvrier... ", art. cit. 
le mieux d'« y trouver son compte " pour reprendre une expression de Burawoy à propos des ouvriers. En effet, ce jeu prévoit un avantage financier pour les salariés qui y trouvent un lien intéressé entre leur engagement et les résultats imposés. II contribue à les rendre indépendants par rapport à l'ensemble du personnel en individualisant leurs relations avec les clients.

Globalement, les managers soutiennent ces jeux dans la limite des intérêts de l'entreprise. Mais, comme dans les usines, l'engagement dans ces jeux contribue à transformer les conflits potentiels entre les salariés et les managers ou les clients, en des conflits entre les salariés, autour des règles et des résultats du jeu. Ces tensions sont favorisées par la reconnaissance que les clients montrent à certains salariés.

Par ailleurs, les salariés peuvent renforcer leur identité au travail en reconfigurant leur position dans la hiérarchie. Dans les deux hôtels, Sherman s'est aperçue que les salariés parlent assez souvent des clients et que les jugements à leur égard peuvent être très contradictoires : parfois, les salariés sont incrédules face aux histoires des clients qu'ils jugent comme une extravagance liée à leur situation financière très favorisée; d'autres fois, ils s'en prennent aux clients qu'ils jugent trop « minables ». Ces commentaires permettent aux salariés de limiter leur subordination vis-à-vis des clients. Les salariés mobilisent, de manière sélective, des grilles de classement (l'intelligence, la moralité, la compétence) qui leur permettent parfois de se sentir comme " supérieurs " aux clients.

De leur côté, les clients cherchent souvent à individualiser leur relation avec les salariés. En ce sens, leur consommation du luxe est ambivalente et contradictoire. Par leurs comportements, ils essaient d'instituer des formes de réciprocité avec les salariés : ils laissent des pourboires, se comparent à d'autres clients impolis, ou encore peuvent juger que les salariés des hôtels ne sont pas les plus défavorisés (" il y a toujours pire »). Cependant, en cherchant implicitement à dépasser l'inégalité sociale de la relation client-salarié, ils la renforcent.

Le travail de Sherman s'appuie sur des données de terrain particulièrement riches qui permettent de donner de la substance à un thème, le luxe, trop souvent traité de manière désincarnée ${ }^{11}$. Son ouvrage décrit le service de luxe et les différentes organisations qui peuvent être mises en place pour le réaliser. II propose une analyse stimulante de l'inter-action entre les clients et les salariés, qui constitue aussi le moment de la consommation-production d'une partie du service de luxe (celui qui comporte notamment l'accompagnement et l'accueil des clients). En revanche, si l'auteure montre, avec intérêt, que ces relations ne sont pas nécessairement conflictuelles, son argumentation en faveur d'un obscurcissement des relations de classes dans des relations individuelles n'est pas complètement convaincante.

Tout d'abord, ses thèses autour de la "banalisation " et de la « normalisation » des relations de classes vécues comme des relations individuelles entre les clients et les salariés ne concernent que les salariés "visibles". Les « invisibles » ont moins d'interactions directes avec les clients ; ils ne peuvent pas élaborer les mêmes stratégies pour " accepter l'exploitation de classe ». Or, ils constituent une part importante du système.

Plus fondamentalement, les classes sociales sont ici simplement évoquées en rapport à la position différente que les salariés et les clients occupent dans la division locale et internationale du travail. Si cela permet de mettre en avant la distance sociale entre les clients et les salariés (qui, étant donné les prix pratiqués dans l'hôtellerie de luxe, paraît aller de soi), une définition plus précise des propriétés sociales, des fractions de classes et des trajectoires aurait, sans doute, permis d'interpréter les différences, les types de rôles ou encore le turnover élevé au sein du personnel ${ }^{12}$. Qu'y a-t-il de commun par exemple entre des femmes de chambre issues de limmigration hispanique ou chinoise et des réceptionnistes d'origine européenne qui, pour certains, viennent réaliser des expériences professionnelles pour des périodes déterminées ${ }^{13}$ ?

La réponse ne peut pas être trouvée seulement à l'intérieur de l'hôtel. Les profils des salariés sont hétérogènes en raison du type de travail qu'ils réalisent - travail en interaction avec les clients ou non -, du secteur de l'organisation dans lequel ils sont employés - réception, étages, restauration ou bar, entretien - mais aussi de leurs propres caractéristiques sociales et de leurs trajectoires de vie. L'observation de l'ensemble du collectif des salariés dans l'hôtel de luxe dévoile un univers professionnel éclaté, traversé par des tensions qui ne peuvent que rendre difficile l'essor chez les salariés d'une prise de conscience d'une condition sociale commune. C'est pourquoi il serait pertinent de se concentrer davantage sur la position de chacun, client ou salarié, dans la division sociale et internationale du travail ${ }^{14}$. Cependant, la nature des interactions entre les salariés et les clients peut être saisie à partir de l'analyse de leur positionnement dans les rapports sociaux de service ${ }^{15}$. Cette relation

11. Lucien Karpik, L'Économie des singularités, Paris, Gallimard, coll. «Bibliothèque des sciences humaines ", 2007.

12. Ce qui tendrait à relativiser la théorie de Sherman sur l'engagement des salariés.

13. D'ailleurs, Sherman ne cache pas que même dans ces hôtels des formes de résistance peuvent exister.

14. Émile Durkheim, De la division du travail social, Paris, PUF, coll. " Quadrige ", 2007 [1893]

15. Jean Gadrey, "Rapports sociaux de service : une autre régulation ", Revue économique, 41(1), 1990, p. 49-70. 
de service doit respecter certains standards « théâtraux » qui contribuent à la définition du service de luxe. Elle est profondément façonnée par les rapports sociaux qui la dépassent. Ici, l'imbrication entre des enjeux économiques et sociaux est particulièrement évidente. Les salariés, surtout ceux qui sont en interaction avec les clients, contribuent à la réussite de la représentation qui constitue aussi le moment de la consommation-production du service de l'accueil et de l'accompagnement des clients, mais leur marge de manœuvre est limitée. La scène sociale sur laquelle ils accomplissent leur service est structurée par le rapport marchand entre l'hôtel et ses clients : elle est très contraignante.

La thèse de l'individualisation des interactions dans la relation de service est donc ambivalente : d'une part elle n'est valable que pour une partie des salariés (les membres du " front office »), d'autre part on pourrait considérer cette mise en scène - "le service théâtral » - non seulement comme une conséquence paradoxale de l'engagement des salariés et des clients dans les interactions mais aussi comme une variable structurale qui contribue à définir l'« ordre de l'interaction ${ }^{16}$ " dans le hall d'un hôtel de luxe et qui dépend du rapport marchand entreprise-clients. Si plusieurs travaux ${ }^{17}$ montrent que l'individualisation des relations client-salarié et la personnalisation du service constituent des conditions nécessaires à la réalisation d'une prestation de service, l'euphémisation des rapports de domination par la mise en scène de relations " amicales " entre les clients et les salariés, dans un contexte où les écarts de classe sont importants et visibles, laisse penser que l'hôtellerie de luxe fonctionne en partie sur le modèle de "l'économie des biens symboliques » proposé par Bourdieu ${ }^{18}$. La production de " l'enchantement »19 propre à ce service de luxe nécessite la mise en place d'un cadre spécifique (infrastructures, etc.) mais aussi l'adhésion, la participation des salariés et des clients dans le type de relations proposées. Ainsi, le luxe serait de pouvoir « faire comme si » le serviteur est un « ami » alors que l'écart social est immense.

16. Erving Goffman, "L'ordre social et l'interaction », in Erving Goffman, Les Moments et leurs hommes, (textes recueillis et présentés par Yves Winkin), Paris, Seuil/Minuit, 1988, p. 95-103.

17. Jean-Pierre Durand et Marie-Christine Le Floch (dir.), La Question du consentement au travail, L'Harmattan, collection «Logiques sociales ", 2006 ; Rémy Caveng, Bertrand Réau et Gérard Rimbert (dir.), Regards sociologiques, 32, 2006.

18. Pierre Bourdieu, "L'économie des biens symboliques ", in Raisons pratiques. Sur la théorie de l'action, Paris, Seuil, 1994.

19. Franck Poupeau et Bertrand Réau, "L'enchantement du monde touristique ", Actes de la recherche en sciences sociales, 170, décembre 2007, p. 4-13. 